\title{
Prevalencia de discapacidad mental en adultos mayores en cinco provincias de Cuba, 2000-2004
}

\author{
Prevalence of physical disability in aged people from five \\ provinces in Cuba, 2000-2004
}

\section{Héctor Héctor D. Bayarre Vea'; Julia S. Pérez Piñero"; J esús Menéndez J iménez" ';Teddy O. Tamargo Barbeito' ;Agustín Morejón Carralerov; Dayamí Díaz GarridoVI ; Arlín González de Piñera Marrero ${ }^{\text {II }}$}

\author{
'Doctor en Ciencias de la Salud. Profesor Titular. Escuela Nacional de Salud Pública. \\ La Habana, Cuba. \\ "Doctora en Ciencias de la Salud. Profesora Auxiliar. Escuela Nacional de Salud \\ Pública. La Habana, Cuba. \\ IIIEspecialista de II Grado en Geriatría. Profesor Auxiliar. Centro de Investigaciones \\ sobre Longevidad, Envejecimiento y Salud. La Habana, Cuba. 'V Máster en Salud \\ Pública. Profesor Auxiliar. Escuela Nacional de Salud Pública. La Habana, Cuba.

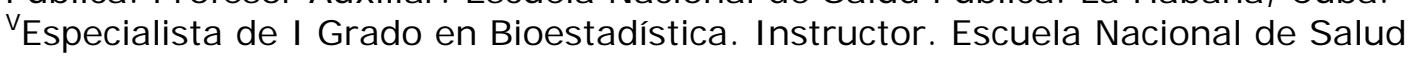 \\ Pública. La Habana, Cuba. \\ ${ }^{V I}$ Especialista de II Grado en Bioestadística. Instructor. Escuela Nacional de Salud \\ Pública. La Habana, Cuba. \\ VIIEspecialista de I Grado en Bioestadística. Escuela Nacional de Salud Pública. La \\ Habana, Cuba.
}

\section{RESUMEN}

Introducción La discapacidad mental en la tercera edad es uno de los problemas más importantes que enfrenta la comunidad científica cubana en la actualidad. Objetivos Estimar la prevalencia de discapacidad mental en adultos mayores de Ciudad de La Habana, Las Tunas, Granma, Holguín y Camagüey, entre los años 2000 y 2004.

Métodos Se realizó un estudio descriptivo de corte transversal, sobre muestras complejas (muestreo por conglomerados polietápico) de adultos mayores seleccionados de forma independiente para cada provincia, cuyos tamaños se determinaron mediante la fórmula de cálculo para estudios descriptivos en poblaciones finitas. Se aplicó el Examen Mínimo del Estado Mental. Se estimaron tasas de prevalencia de discapacidad mental, globales y específicas. El procesamiento de los datos se efectuó mediante programas computarizados.

Resultados La discapacidad mental varió entre 4,5\% (Holguín) y 21,5\% 
(Granma) Se incrementó con la edad, desde 0,3\%, para los adultos mayores de 60-69 años que residen en Holguín, hasta $56,3 \%$ en los de 80 y más de Granma; predominó en mujeres de todas las provincias, con tasas que alcanzaron el $20 \%$, excepto para Las Tunas $(13,5 \%)$ y Holguín $(5,8 \%)$. Las principales afectaciones en la esfera mental se relacionaron con el cálculo y el recuerdo tanto para el grupo con discapacidad como para los que no la tenían, independientemente de la provincia.

Conclusiones La prevalencia de discapacidad mental en las provincias investigadas varía desde un bajo nivel en Holguín hasta uno alto en Granma. Los mayores niveles de discapacidad mental encontrados en todos los territorios se corresponde con la edad avanzada, el sexo femenino, la baja escolaridad y la desocupación. Las afectaciones mentales más frecuentes de los adultos mayores investigados se presenta en el cálculo y el recuerdo; ello debe tomarse en cuenta en cualquier estrategia de intervención que se diseñe al efecto.

Palabras clave: Adulto mayor, discapacidad mental, prevalencia, Cuba.

\section{ABSTRACTS}

Introduction Mental disability in the aged people is one of the most important problems that the Cuban scientific community currently faces.

Objectives To estimate the prevalence of mental disability in the aged people from the City of Havana, Las Tunas, Gramma, Holguín and Camagüey provinces from 2000 and 2004.

Methods A cross-sectional descriptive study of complex samples (multi-stage cluster sampling) of randomly selected aged people from each of the above provinces, whose size was estimated through a calculation formula for descriptive studies in finite populations, was conducted. The Minimal Mental Status Examination was applied. Global and specific mental disability prevalence rates were estimated. Data processing was computerized.

Results Mental disability ranged from $4.5 \%$ (Holguín province) to $21.5 \%$ (Gramma province). It increased with the age, from $0.3 \%$ in $60-69$ y age group dwelling in Holguín to $56.3 \%$ in the 80 years and over group in Las Tunas and it was predominant in females from all these provinces, with $20 \%$ rates, except for Las Tunas $(13.5 \%)$ and Holguin $(5.8 \%)$. The main problems in the mental status were related to calculating and remembering, both in the disabled group and in the nondisabled group regardless of the province.

Conclusions Mental disability prevalence changes in the studied provinces, ranging from low rates in Holguin provinces to high rates in Gramma province. The highest rates of mental disability found in all these territories were associated with old age, female, low education and inactivity. The most frequent mental disturbances were calculation and remembering; all this should be taken into account for any intervention strategy to be designed to this end.

Key words: Older people, physical disability, prevalence, Cuba.

\section{NTRODUCCI ÓN}


El estudio de los procesos de transición demográfica y epidemiológica ha cobrado interés en las últimas décadas, entre otras razones porque el envejecimiento poblacional iniciado en Europa -en países con transición temprana- se ha extendido a otras regiones del mundo, a la vez que ha incrementado su velocidad. Paralelo a ello se han producido cambios en los patrones de morbilidad y mortalidad, que han modificado la demanda de los servicios sociales y de salud. ${ }^{1}$

La prolongación de la esperanza de vida ha sido un anhelo de la humanidad. En este sentido, el envejecimiento puede ser considerado un logro en el que mucho ha aportado el desarrollo científico técnico alcanzado. ${ }^{2}$ Sin embargo, la longevidad está produciendo retos sin precedentes a los responsables de la elaboración de políticas en general y a los ciudadanos en particular, pues se deberán mantener los niveles de seguridad social y económica, y la oferta de servicios de salud de calidad a un segmento de la población que por su avanzada edad, se encuentra en desventaja para afrontar las exigencias sociales de la contemporaneidad. ${ }^{3}$

El desarrollo de las ciencias en general y de las ciencias médicas y de la salud en particular, ha demostrado que lo más importante no es que haya un aumento de la expectativa de vida, sino que sea posible envejecer manteniendo una calidad de vida adecuada. ${ }^{4}$ Desde esta perspectiva, numerosas investigaciones gerontológicas centran su atención en la evaluación de la calidad de la vida de los adultos mayores, al considerar esta como un indicador de envejecimiento satisfactorio, pues adiciona a la supervivencia, información sobre el bienestar a un menor costo social. $^{2}$

En el estudio de la calidad de vida en la población anciana hay que tener en cuenta aspectos tales como el estado de salud, la determinación de factores de riesgo de discapacidad, la predicción de discapacidades, el bienestar subjetivo y el estado funcional de este grupo poblacional. ${ }^{2}$

Las discapacidades físicas y mentales, íntimamente relacionadas con el incremento de la expectativa de vida, se considera entre los problemas más graves a enfrentar en la actualidad. ${ }^{5,6}$ Su evaluación es difícil, ya que estas son consecuencia de interacciones entre alteraciones estructurales y funcionales del organismo, unido a una multitud de factores psicológicos y sociales.

Para el estudio de la esfera mental se han desarrollado varios instrumentos que evalúan el deterioro cognitivo, entre los que destaca el Examen Mínimo del Estado Mental (EMEM) de Folstein, Folstein y Mc Hugh (1975), con el que se logra un cuestionario para el pesquisaje del deterioro cognoscitivo o la caracterización del funcionamiento en esta esfera. Este instrumento, de amplio uso en la investigación en grupos comunitarios, abarca las áreas de la orientación, el registro, la atención, el cálculo, el recuerdo, el lenguaje y la construcción vídeo espacial. ${ }^{7,8}$

El estudio del deterioro cognitivo, incluido el de las demencias, ha sido objeto de interés en investigadores de diversas latitudes. Ello parece estar dado no sólo por su magnitud, sino por la repercusión de este en el ámbito individual, familiar y social.

Varios son los estudios que, sobre la discapacidad mental en el anciano, se han realizado en Cuba en la última década por un grupo de investigadores de la Escuela Nacional de Salud Pública (ENSAP) y del Centro I beroamericano para la Tercera Edad (CITED); en ellos se estimó la prevalencia de discapacidad mental en ancianos del municipio Playa. ${ }^{1}$ Se efectuaron además, dos investigaciones en municipios de la provincia Las Tunas. ${ }^{9}$ Estos estudios han permitido estimar la prevalencia de la discapacidad mental, entre 13 y 26,2 por ciento. 
A pesar de la información brindada por los estudios antes mencionados, es menester continuar realizando investigaciones en otros contextos del país y en los niveles provincial y nacional, con vistas a brindar datos útiles para la gestión en salud en estos ámbitos, al constituir evidencia científica actualizada sobre la magnitud del problema, las principales variables relacionadas con su aparición y su comportamiento temporal.

Como elemento favorecedor para el desarrollo de estos estudios se dispone del Programa de Atención al Adulto Mayor que es uno de los cuatro priorizados del Ministerio de Salud Pública de $\mathrm{Cuba}^{10}$ y que incluye, en todos los municipios del país, los gabinetes gerontológicos, integrados por un equipo multidisciplinario, que tiene dentro de sus propósitos una atención integral al adulto mayor para la reducción de sus discapacidades. A esto se adiciona la creación de un Programa Ramal de Investigaciones para este grupo poblacional, entre cuyas líneas de investigación aparecen las discapacidades físicas y mentales, por lo que la investigación sobre este tema constituye un instrumento de gran utilidad.

Por otra parte, las condiciones materiales de vida han sido consideradas entre los principales indicadores a incluir en la evaluación del componente objetivo de la calidad de vida. Tal consideración ha devenido en importante razón para su utilización en la conformación de los grupos a investigar.

Dos estudios realizados por Sánchez Labrada, Astraín Rodríguez y Alonso Alomá, Astraín Rodríguez, aún no publicados, clasificaron a las povincias de Cuba según condiciones de vida. Para ello se consideraron como unidades de análisis los municipios. Entre las provincias, Ciudad de La Habana ocupa el lugar cimero, mientras Las Tunas y Granma aparecen entre las últimas; Camagüey ocupa una posición intermedia y Holguín tiene un comportamiento heterogéneo en sus condiciones de vida. Estos datos coinciden con los aportados por la investigación relativa al Índice de Desarrollo Humano en Cuba. ${ }^{11}$ De ahí que, partiendo del "impacto" reconocido de las condiciones de vida sobre la salud y la calidad de la vida de la población, resulte interesante estudiar discapacidades físicas y mentales en la población adulta mayor de estas provincias.

Teniendo en cuenta los argumentos anteriores, se decidió realizar esta investigación, con el propósito de aumentar el caudal de conocimientos acerca de la prevalencia de la discapacidad mental en las personas de edad avanzada que aporten elementos para el diseño de estrategias de intervención a escala individual, familiar y comunitaria en la población de personas de edad de Ciudad de La Habana, Camagüey, Las Tunas, Granma y Holguín, además, brindar información válida para la planificación de recursos y servicios, lo que puede influir sobre la organización de los servicios, la dirección y el control; así como sobre la aplicación de técnicas gerenciales, entre ellas, la toma de decisiones y el establecimiento de prioridades. Esto sería posible si con el estudio se logra responder la siguiente interrogante:

¿Cuál es la magnitud de la discapacidad mental global y según variables seleccionadas en la población anciana de las provincias Ciudad de La Habana, Camagüey, Las Tunas, Granma y Holguín?

\section{MÉTODOS}


El trabajo clasifica como estudio descriptivo, de corte transversal, de prevalencia, realizado en la población de 60 años y más que residen en: Ciudad de La Habana, Las Tunas, Granma, Holguín y Camagüey, entre el año 2000 y el 2004.

El estudio incluyó las variables siguientes: provincia, discapacidad mental, sexo, edad, escolaridad, ocupación, orientación en tiempo y espacio, registro, cálculo y atención, recuerdo y lenguaje.

\section{Aspectos éticos}

Antes de ser incluidos en el estudio se le solicitó a cada adulto mayor, o en su defecto a un familiar (en caso de deterioro cognitivo importante), su consentimiento (anexo). Se explicaron los objetivos del estudio y la importancia de su participación. Se les garantizó la confidencialidad de la información que ellos aportarían y la posibilidad de abandonar el estudio si así lo deseasen, sin repercusión alguna ante la necesidad de atención médica posterior.

\section{Técnicas y procedimientos}

El universo de cada estudio, estuvo constituido por todos los ancianos que residían en la provincia Ciudad de La Habana y Las Tunas, durante el primer semestre del 2000; Granma y Holguín en el primer semestre de 2002 y Camagüey en el primer semestre de 2004. El tamaño muestral se determinó, en cada caso, a través de la fórmula para la determinación del tamaño mínimo necesario en estudios descriptivos. ${ }^{12}$ De la Ciudad de La Habana se escogió una muestra de 728 ancianos, de Las Tunas se escogieron 698, de Granma 700, Holguín 701 y de Camagüey 866.

Para la selección definitiva, se utilizó como técnica muestral el Muestreo Estratificado Polietápico (MEP). ${ }^{12}$

Para la recogida de la información se aplicaron los siguientes instrumentos: Examen Mínimo del Estado Mental (EMEM), para evaluar la esfera mental, y una encuesta para la recogida de información de variables sociodemográficas. El EMEN es un instrumento de amplio uso en la investigación gerontológica y cuenta con los atributos de fiabilidad y validez requeridos para su uso.

La aplicación de los instrumentos se realizó por un grupo de estudiantes de excepcional rendimiento, por los médicos de la familia de los consultorios seleccionados que se motivaron con la investigación y por residentes de tercer año de la especialización en bioestadística. Se realizó un adiestramiento previo a los entrevistadores que consistió en un seminario impartido por un psicólogo del CITED. Con el objetivo de asegurar la recogida de la información, se realizó un control de la calidad al $10 \%$ de las entrevistas, seleccionadas al azar.

\section{Técnicas de procesamiento y análisis}

El tratamiento de los datos se realizó mediante programas computarizados. Se realizó la estimación puntual y por intervalo de la tasa global y específica de discapacidad mental. Con la finalidad de controlar el efecto de la estructura poblacional por edad y sexo, se realizó la tipificación de tasas por el método directo. Se escogió como población tipo la suma de las muestras de las provincias del estudio, luego se calcularon, para cada muestra, las discapacidades esperadas por variable; para ello se multiplicaron las tasas específicas por la población tipo de la categoría correspondiente, en cada caso. Finalmente, se obtuvieron las tasas 
tipificadas al dividir la suma de las discapacidades esperadas por el total de la población tipo. Los resultados se presentan en tablas y gráficos. estadísticos.

\section{RESULTADOS}

La estimación de la discapacidad mental varió desde 4,5\% para Holguín, hasta $21,5 \%$ para Granma, constituyendo la primera, la única provincia de las estudiadas con cifra inferior a $10 \%$. La estrechez de la mayoría de los intervalos estimados, expresa la precisión de esta estimación (figura).

Para el control de la posible influencia de las estructuras por edad y sexo de las poblaciones estudiadas, sobre las tasas de discapacidad mental estimadas, se tipificó por el método directo. Al controlar la edad se observó una importante reducción de la discapacidad mental para Ciudad de La Habana (de 18,8 a 12,0 \%) y discretos cambios en las restantes provincias. Sin embargo, aún controlando esta variable, los niveles de discapacidad mental estimados para Ciudad de La Habana fueron superiores a los de Holguín (tabla 1).

Al controlar el sexo, no se obtuvieron cambios importantes en los niveles de la discapacidad estudiada, lo que habla a favor de una influencia escasa o nula de esta variable sobre las diferencias constatadas en las tasas de discapacidad física y mental; aunque el resultado pudiese estar influido por el efecto de otras variables que no se controlaron con este procedimiento (tabla 1).

Para la población investigada de Ciudad de La Habana se observó que en los discapacitados mentales el $93,4 \%$, el $87,6 \%$ y el 77,3 \% tuvieron alteraciones en el cálculo y la atención, el recuerdo y la orientación respectivamente, mientras que en los no discapacitados el 44,1 \% presentó problemas con el cálculo y la atención, con un número de afectados muy inferior en los restantes procesos cognitivos (tabla 2).

En los gerontes de Camagüey se observó que para los discapacitados mentales el $97,8 \%, 94,2 \%$ y 82,7 \% tenían afectaciones del cálculo y atención, el recuerdo, y la orientación respectivamente; mientras que entre los no discapacitados el 55,8 \% y el $46,6 \%$ ya mostraban alteraciones en el recuerdo y el cálculo y la atención.

En los investigados de Las Tunas se apreció que entre los discapacitados mentales, los más afectados fueron el cálculo y la atención con 90,2 \% y el recuerdo con 67,4 $\%$, lo que también ocurrió para los no discapacitados pero en menor cuantía.

En Granma se obtuvo una mayor afectación entre los discapacitados mentales del recuerdo, el cálculo y la atención y el registro, con el $77,0 \%$, el $64,9 \%$ y el 51,4 $\%$ respectivamente, en tanto que entre los que no presentaban un deterioro cognitivo importante, el 33,9 \% tuvo dificultades en el cálculo y la atención.

Los resultados de la exploración del estado mental en los adultos mayores de Holguín mostró las mayores afectaciones en los discapacitados mentales en la realización del cálculo y la atención, con el el 93,5\%, mientras el 90,3\% tenía alteraciones del recuerdo y la orientación. Un escaso número de los no discapacitados presentaba alteraciones en alguna de las dimensiones investigadas.

Llama la atención que los trastornos de la memoria no constituyen las alteraciones más frecuentes en ninguna de las provincias investigadas. 
La prevalencia de discapacidad mental según edad y provincia se incrementó a medida que aumentó la edad. El nivel de discapacidad en Ciudad de La Habana, varió desde 5,2 \% (3,8_6,6\%) en los viejos-jovenes, hasta 51,2\% $\left(40,4 \_62,0 \%\right)$ para el grupo de 80 y más; en Camagüey desde 4,8 \% (2,7- 6,9\%) hasta 50,0 \% $(42,3-57,7 \%)$. Las tasas de discapacidad para Las Tunas oscilaron entre $5,8 \%$ $(1,9-9,6 \%)$ y $42,0 \%(9,5-74,6 \%)$. En Granma la discapacidad se movió entre 7,2 $\%(2,7-11,7 \%)$ y $56,3 \%(45,8-66,7 \%)$. En Holguín las cifras variaron desde 0,3 $\%(0,0-0,8 \%)$ hasta $18,1 \%(0,6-35,7 \%)$ (tabla 3 ).

En la tabla 4 se observa un ligero predominio de este tipo de discapacidad para el sexo femenino, que se hace más evidente en Camagüey con una tasa de $23,2 \%$ vs $9,6 \%$ en el sexo masculino y Ciudad de La Habana con una tasa de $21,0 \%$ vs 15,1 $\%$ para el masculino.

En la tabla 5 se aprecia que en todas las provincias los mayores niveles de discapacidad mental se registraron en la categoría desocupados, con $21,8 \%$ en Granma, 19,9\% para los ancianos de Ciudad de La Habana, y 16,9\% para los de Camagüey, provincias en las que se registraron los niveles más altos para esta categoría.

Para todas las provincias, la discapacidad mental tendió a disminuir a medida que aumentó la escolaridad. En Ciudad de La Habana hay una reducción de 66,1 \% en el grupo de analfabetos a $0,0 \%$ en el grupo de universidad concluida, mientras que en Camagüey se redujeron los niveles de este tipo de discapacidad de $61,2 \%$ a 3,3\%. Para Las Tunas esta se redujo de 68,0 a 0,0 \%. En Granma la reducción fue de $72,2 \%$ a $7,1 \%$ y en Holguín disminuyó de $28,1 \%$ a 0 \% (tabla 6 ).

\section{DISCUSIÓN}

La prevalencia de discapacidad mental fue ligeramente inferior a la encontrada por Tello, ${ }^{9}$ y superior a la de un estudio previo realizado por un grupo de los autores de este trabajo, ${ }^{3}$ con excepción de la encontrada en la investigación de Holguín, lo que tal vez esté relacionado con las características de la población estudiada.

En investigación reciente realizada por Gribsby, ${ }^{13}$ se encuentra que uno de cada tres ancianos está afectado por un deterioro cognitivo ligero, mientras que uno de cada seis tiene un deterioro cognitivo de moderado a grave. Otro estudio realizado por Artero ${ }^{14}$ encuentra niveles de discapacidad mental superiores a los informados por Gribsby.

Los resultados encontrados en la aplicación del EMEM, fueron similares a los de otras investigaciones, ${ }^{9,14-16}$ donde se demuestra que el cálculo, la atención y el recuerdo son las funciones mentales que primero se ven afectadas en las personas de edad avanzada como se refirió anteriormente. ${ }^{17}$ En todos, la memoria, aunque estuvo entre las esferas más afectadas, no ocupó el lugar cimero como era de esperar, lo que pudiera relacionarse con la escolaridad. La escolaridad baja puede devenir en confusor de resultados. ${ }^{18,19}$ Las investigaciones más recientes acerca de los efectos del envejecimiento sobre la memoria señalan que las causas por las que se producen estos trastornos son complejas, existiendo una base biológica (cambios o pérdidas de células cerebrales) y factores psicosociales, probablemente de mayor importancia que las limitaciones somáticas, tales como nivel bajo de inteligencia, falta de hábito para recordar por tener una vida rutinaria y falta de motivación, entre otros. 
En esta investigación se observó un incremento del riesgo de discapacidad mental con la edad, al igual que en otros estudios ${ }^{9,13,20,21}$ y sin grandes diferencias entre los territorios investigados. Se plantea que con los años se altera la percepción, el razonamiento inductivo, la simbolización, así como la inteligencia verbal y el coeficiente de inteligencia va declinando. En otras investigaciones, ${ }^{15,22,23}$ se hace referencia a los cambios que se producen en la memoria con el incremento de la edad. Es de suponer la declinación de las capacidades mentales con la edad, debido a que el envejecimiento provoca deterioro biológico y funcional del individuo.

El sexo femenino al igual que en otras poblaciones de ancianos ${ }^{9}$ presentó una alta prevalencia de discapacidad mental. Se han formulado varias hipótesis al respecto. Algunos autores plantean que la disminución de los niveles de estrógeno que ocurre en la posmenopausia puede incrementar el deterioro cognitivo, pues estos suelen tener un papel protector (esta hipótesis unicausal no es plausible al contrastarla con el sexo masculino en el que existen bajos niveles de estrógenos durante toda la vida). ${ }^{2}$ Otras afirmaciones parecen ser más creíbles, al involucrar factores sociales; así, se aduce que la mujer se ha dedicado a las labores domésticas y al rol reproductivo, con menores posibilidades de desarrollo de trabajos intelectuales, que demandan razonamiento lógico y ello pudiera estar contribuyendo a que la anciana sea más vulnerable a la disminución o pérdida manifiesta de las aptitudes intelectuales.

La prevalencia de discapacidad mental según la ocupación se comportó muy similar entre las provincias estudiadas y acorde a otros autores. ${ }^{2,9}$ Lo anterior pudiera estar relacionado más con una pérdida de las habilidades y con la falta de entrenamiento que provoca la desocupación, que con el deterioro biológico.

Los resultados obtenidos en la discapacidad mental según escolaridad coinciden con otras informaciones. ${ }^{2,}$ 9, 15, 16,23 Estos resultados permiten inferir que a medida que el anciano tiene menor nivel escolar, se ven limitadas sus motivaciones e intereses, lo que puede afectar la calidad de su intelecto y su capacidad mental en general. Por otra parte la forma en que se explora la capacidad mental mediante el EMEM, puede colocar en posiciones desventajosas a las personas de edad subescolarizadas, ya que algunas tareas tienen que ver con el dominio de operaciones aritméticas y la utilización del lenguaje escrito. A pesar de esto, el EMEM sigue siendo un método extendido en todo el mundo para evaluar la esfera cognoscitiva del anciano.

Se uede concluiur que la prevalencia de discapacidad mental en las provincias investigadas varía desde un bajo nivel en Holguín hasta un alto en Granma. Los mayores niveles de discapacidad mental encontrados en todos los territorios se corresponden con la edad avanzada, el sexo femenino, la baja escolaridad y la desocupación. Las afectaciones mentales más frecuentes de los adultos mayores investigados se presentan en el cálculo y el recuerdo; ello debe tomarse en cuenta en cualquier estrategia de intervención que se diseñe al efecto.

\section{REFERENCI AS BI BLI OGRÁFICAS}

1. Bayarre Vea HD, Pérez Piñero J, Menéndez Jiménez J. Las transiciones demográfica y epidemiológica y la calidad de vida objetiva en la tercera edad. Rev Gerol nfo [serie en Internet]. 2006 [citado Ene 2008]; 1(3). Disponible en: http://www. sld.cu/sitios/gericuba/temas/php?idv =10393. 
2. Bayarre Vea HD. Prevalencia y factores de riesgo de discapacidad en anciano. Ciudad de La Habana y Las Tunas, 2000 [Tesis]. La Habana: Escuela nacional de salud Pública; 2003. Disponible en:

http://www.sld.cu/galerias/pdf/sitios/revsalud/prevalencia_y_factores_de_riesgo.p df.

3. Hernández Castellón R. Estudio del envejecimiento de la población. En: CEDEM, editor. Perspectivas y escenarios de la población y los recursos humanos de Cuba y sus implicaciones económicas y sociales entre el año 2000 y 2050. La Habana:

CEDEM; 2000.p.374-418.

4. Vergara E, Fernández JA, Morales F, Cabañas LA. Influencia del círculo de auelos sobre la salud. Estudio comparativo entre un grupo de integrantes y un grupo control. Rev Cubana Med Gen Integr. 1994; 10:321-5.

5. Bayarre Vea HD, Fernández Fente A, Trujillo Gras O, Menéndez Jiménez J. Prevalencia de discapacidad física en ancianos del municipio Playa. 1996 (Parte I). Rev Cubana Salud Pública. 1999; 25 (1): 16- 29.

6. Bayarre Vea HD, Fernández Fente A, Trujillo Gras O, Menéndez J iménez J. Prevalencia de discapacidad mental en ancianos del municipio Playa. 1996 (Parte II). Rev Cubana Salud Pública. 1999; 25 (1):30-8.

7. Folstein M, Folstein S, Mc Hugh PR. Minimental state: a practical method for grading the cognitive state patients for the clinicians. J Psichiatric Res. 1975; 12: 189-98.

8. Folstein $\mathrm{M}$, Anthony JC, Parhad $\mathrm{Y}$. The meaning of cognitive impairment in the elderly. J Am Geriat Soc. 1988; 18: 716-26.

9. Bayarre Vea HD, Tello Velázquez Y, Hérnández Pérez Y, Herrera Domínguez $H$, Selva AL. Prevalencia de discapacidad mental en ancianos. Municipios Amancio Rodríguez y Jobabo. 1999. Rev Cubana Salud Pública. 2002;28(2):100-11.

10. Ministerio de Salud Pública (Cuba). Programa Nacional del Adulto Mayor. La Habana: MINSAP; 1996.

11. Investigación sobre Desarrollo Humano en Cuba. 1996. La Habana: Caguayo; 1997.

12. Silva Ayçaguer LC. Diseño razonado de muestras y captación de datos para la investigación sanitaria. Madrid: Díaz de Santos; 2000.

13. Gribsby J, Kaye K, Shetterly SM, Baxter J, Morgenstern NE, Hamman RF. Prevalence of disorders of executive cognitive functioning among the elderly: findings from the San Luis Valley. Health and Aging Study. Health Aging. 2002; 21(5):213-20.

14. Artero S, Touchon J, Ritchie K. Disability and mild impairment: a longitudinal population- based study. Int J Geriatr Psychiatry. 2001;16(11):1092-7.

15. Lorsbach TC, Simpson GB. Age differences in the rate of processing in shortterm memory. J Gerontol. 1997; 39: 315-21. 
16. Ligkt LL, Sing A. Implicit and explicit memory in young and elders adults. J Exp Psychol. 1997; 13:531- 41.

17. López JM, Sánchez M. Fragilidad cognitiva en el anciano: revisión de sus causas. Rev Esp Geriatr Gerontol. 1997; 32: 7-14.

18. Belloch FA. Aspectos psicológicos del envejecimiento. En: Anzola E, editor. La atención del anciano: un desafío de años 90. Washington, D.C.: OPS; 1994. p.1229. Publicación Científica No. 546

19. Mc Ghie A, Chapman J, Lauson J. Changes in immedite memory with age. Br J Psychol. 1965;56:69-75.

20. Owens WA. Age and mental abilities: a second adult follow-up. Educ Psychol. 1996; (126): 150- 61.

21. Shaick $C$. The effect and time cohort differences upon age: changes in cognitive behavior. Multivariate Behavioral Res. 1998:259-94.

22. Craik $\mathrm{FI}$. On the tansfer information from temporaly to permanent memory (London). Phil Trans R Doc. 1999;302: 341-59.

23. Borke DM, Light LL. Memory and aging: the rol of retinal processes. Psichol Bull. 1998; 90:513-46.

Recibido: 7 de enero de 2008.

Aprobado: 10 de marzo de 2008.

Héctor D. Bayarre Vea. Calle 80 No. 1509 e/ 15 y 17. Playa. La Habana, Cuba. Emails: hbayarre@infomed.sld.cu, hdbayarre@yahoo.com

\section{Anexo}

Consentimiento de participación

La investigación de las discapacidades física y mental en la tercera edad es de suma importancia por la frecuencia con que se presenta, afectando la salud de este segmento poblacional. El propósito del estudio es aportar datos sobre el nivel de las discapacidades física y mental, así como sobre los factores de riesgo de la discapacidad física en las provincias del estudio, que puedan servir para mejorar la calidad de la atención de esta población y repercutir en la calidad de vida de los adultos mayores de estos territorios. El presente documento de consentimiento, entregado por uno de los investigadores del proyecto titulado "Prevalencia yfactores de resgo de dscapacidad en ancianos. Ciudad de La Habana, Camagüey, Las Tunas, 
Granma y Holguín. 2000-2004", previa consulta oral, es parte del protocolo de investigación que ha sido aprobado por el cmité de ética correspondiente.

Yo ejerciendo mi libre poder de elección y mi voluntad expresa, por este medio, doy mi consentimiento para responder los instrumentos del estudio.

He tenido tiempo suficiente para decidir mi participación, sin sufrir presión alguna y sin temor a represalias en caso de rechazar la propuesta.

También se me ha explicado que la información que brinde es de carácter confidencial y que no serán divulgados ni publicados, ni mi identidad, ni los detalles personales.

Estoy consciente de mi derecho a no responder cualquier pregunta que considere indiscreta, sin tener que dar razones para esto y sin que afecte las relaciones con el equipo médico, por lo que tendré derecho a continuar recibiendo la atención médica establecida, aún si me niego a participar en el estudio.

Para que así conste firmo el presente consentimiento a los días del mes de 200

Firma

Encuesta para medir la discapacidad mental y factores asociados a ésta.

Datos generales.

Nombre: Fecha:

Edad: Ocupación: Escolaridad:

Policlínico: Consultorio:

Municipio: Provincia:

\begin{tabular}{|c|c|}
\hline $\begin{array}{l}\text { I. Orientación } \\
\text { (máximo } 10 \\
\text { puntos) } \\
\text { Interrogar al } \\
\text { anciano sobre } \\
\text { fecha de hoy, } \\
\text { estación del año, } \\
\text { etc. Interrogar al } \\
\text { anciano }\end{array}$ & 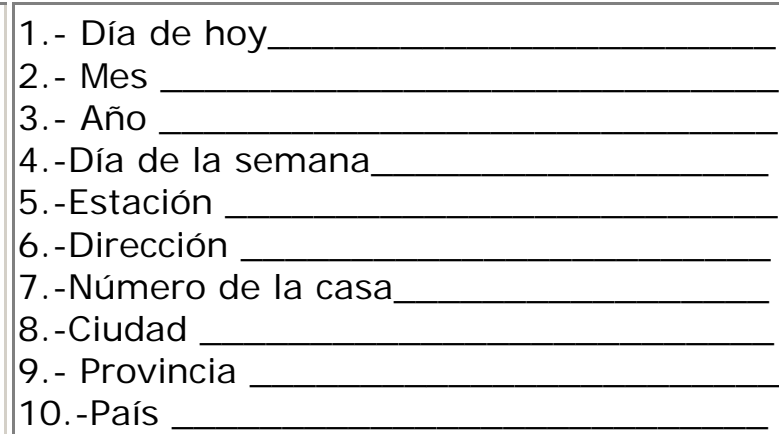 \\
\hline
\end{tabular}




\begin{tabular}{|c|c|}
\hline $\begin{array}{l}\text { acerca del } \\
\text { nombre de la } \\
\text { calle dónde } \\
\text { habita o } \\
\text { vive, número de } \\
\text { su casa. } \\
\text { ¿En qué ciudad } \\
\text { estamos? } \\
\text { ¿Qué provincia? } \\
\text { ¿Qué país? }\end{array}$ & \\
\hline $\begin{array}{l}\text { II. Registro: } \\
\text { (máximo } 3 \\
\text { puntos) } \\
\text { Explorar la } \\
\text { capacidad de } \\
\text { memoria. Repetir } \\
\text { despacio las } \\
\text { palabras: PELOTA/ } \\
\text { BANDERA/ } \\
\text { ARBOL. } \\
\text { Repartirlas hasta } \\
6 \text { veces para que } \\
\text { el } \\
\text { anciano las } \\
\text { aprenda. } \\
\text { Registrar los } \\
\text { intentos. }\end{array}$ & 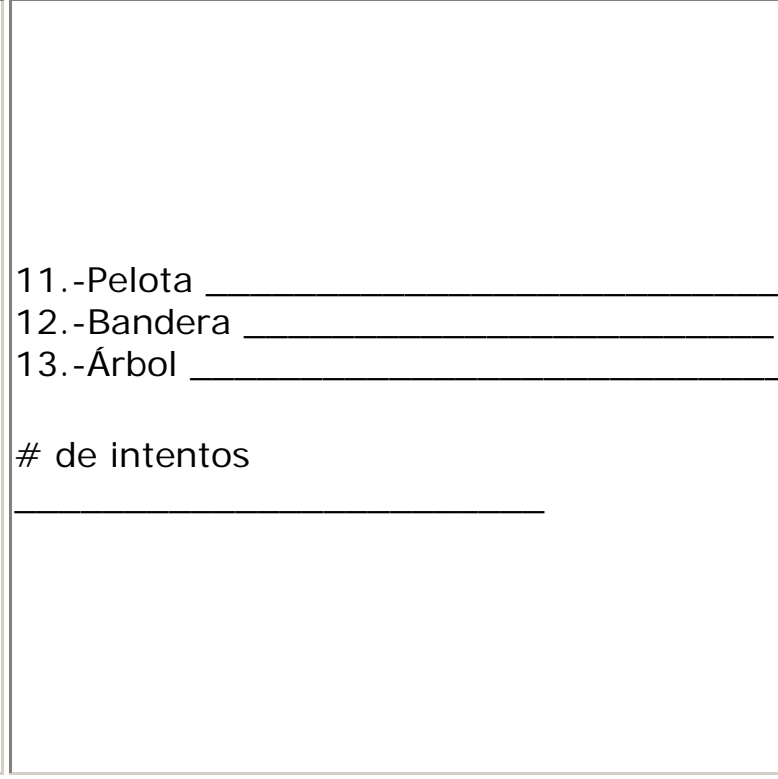 \\
\hline $\begin{array}{l}\text { III. Cálculo y } \\
\text { Atención: } \\
\text { (máximo } 5 \\
\text { puntos) } \\
\text { Contar desde } 100 \\
\text { hasta abajo } \\
\text { restando } 7 . \\
\text { Parar después de } \\
5 \text { respuestas: } \\
\text { (93, 86, 79, } \\
72,65 \text { ). Dar un } \\
\text { punto por cada } \\
\text { respuesta } \\
\text { correcta. Si el } \\
\text { sujeto no puede } \\
\text { realizar las } \\
\text { tareas, debe } \\
\text { deletrear la } \\
\text { palabra MUNDo } \\
\text { de atrás hacia } \\
\text { delante. Dar un } \\
\text { punto por cada } \\
\text { letra bien } \\
\text { ubicada. Ej.: } \\
\text { ODNUM=5 / } \\
\text { ODMUN=3 }\end{array}$ & 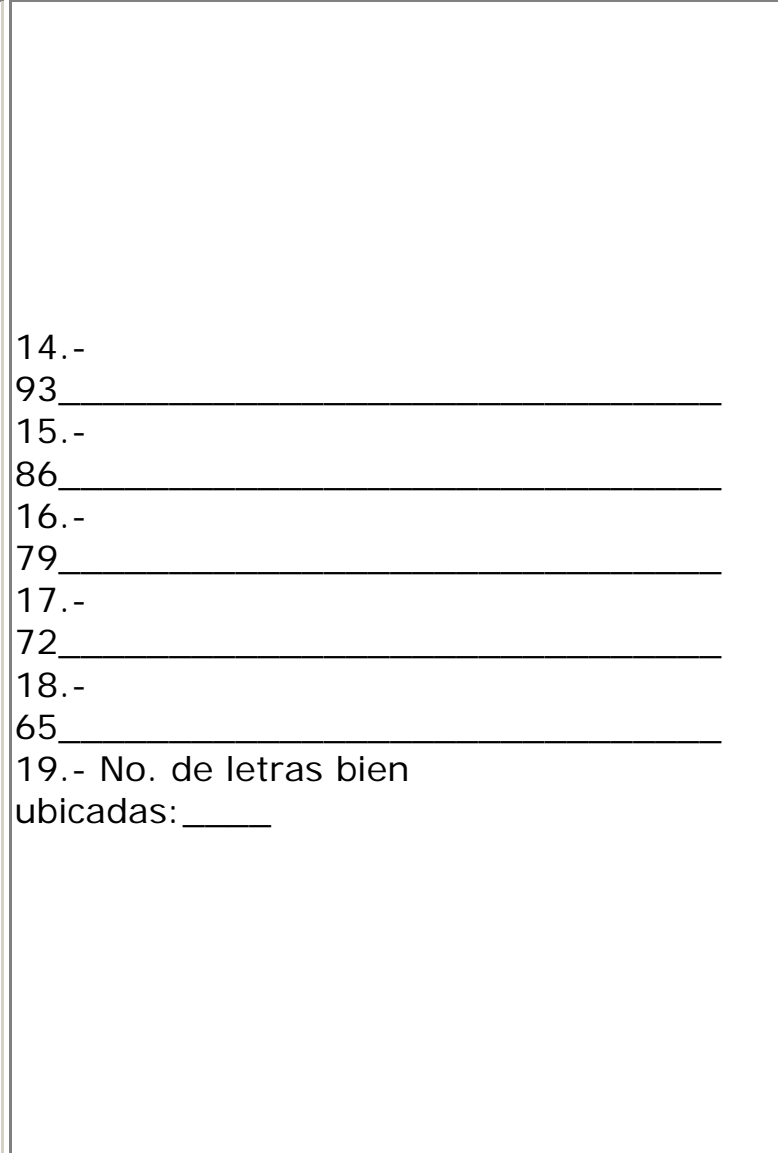 \\
\hline
\end{tabular}




\begin{tabular}{|c|c|}
\hline $\begin{array}{l}\text { IV. Recuerdo: } \\
\text { (máximo } 3 \\
\text { puntos) } \\
\text { Preguntar por las } \\
\text { tres palabras } \\
\text { repetidas } \\
\text { previamente. Un } \\
\text { punto por cada } \\
\text { respuesta } \\
\text { correcta. }\end{array}$ & 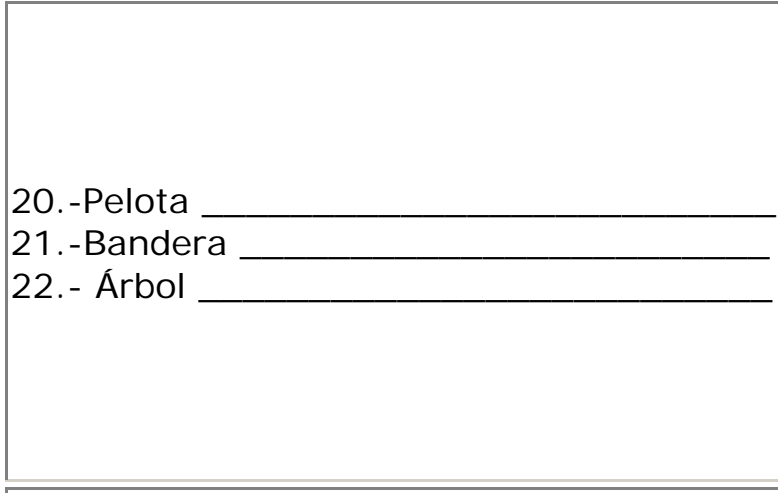 \\
\hline $\begin{array}{l}\text { V. Lenguaje: } \\
\text { ( máximo } 9 \\
\text { puntos ) } \\
\text { - Mostrar al } \\
\text { anciano un reloj y } \\
\text { preguntarle: } \\
\text { "¿Qué es esto?" } \\
\text { Repetir lo mismo } \\
\text { para el lápiz. Un } \\
\text { punto por } \\
\text { respuesta } \\
\text { correcta. } \\
\text { - Repetir la frase: } \\
\text { "Ni sí, ni no, ni } \\
\text { peros". } \\
\text { - Realizar una } \\
\text { orden en } 3 \\
\text { tiempos (Tomar } \\
\text { un papel con la } \\
\text { mano derecha, } \\
\text { doblarlo por la } \\
\text { mitad y ponerlo } \\
\text { en el piso). } \\
\text { - Leer y } \\
\text { obedecer: } \\
\text { ClERRE LOS } \\
\text { OJ OS. } \\
\text { - Escritura: } \\
\text { Orientar al sujeto } \\
\text { para que escriba } \\
\text { una frase u } \\
\text { oración. } \\
\text { - Copiar un } \\
\text { dibujo del Test de } \\
\text { Bender. }\end{array}$ & 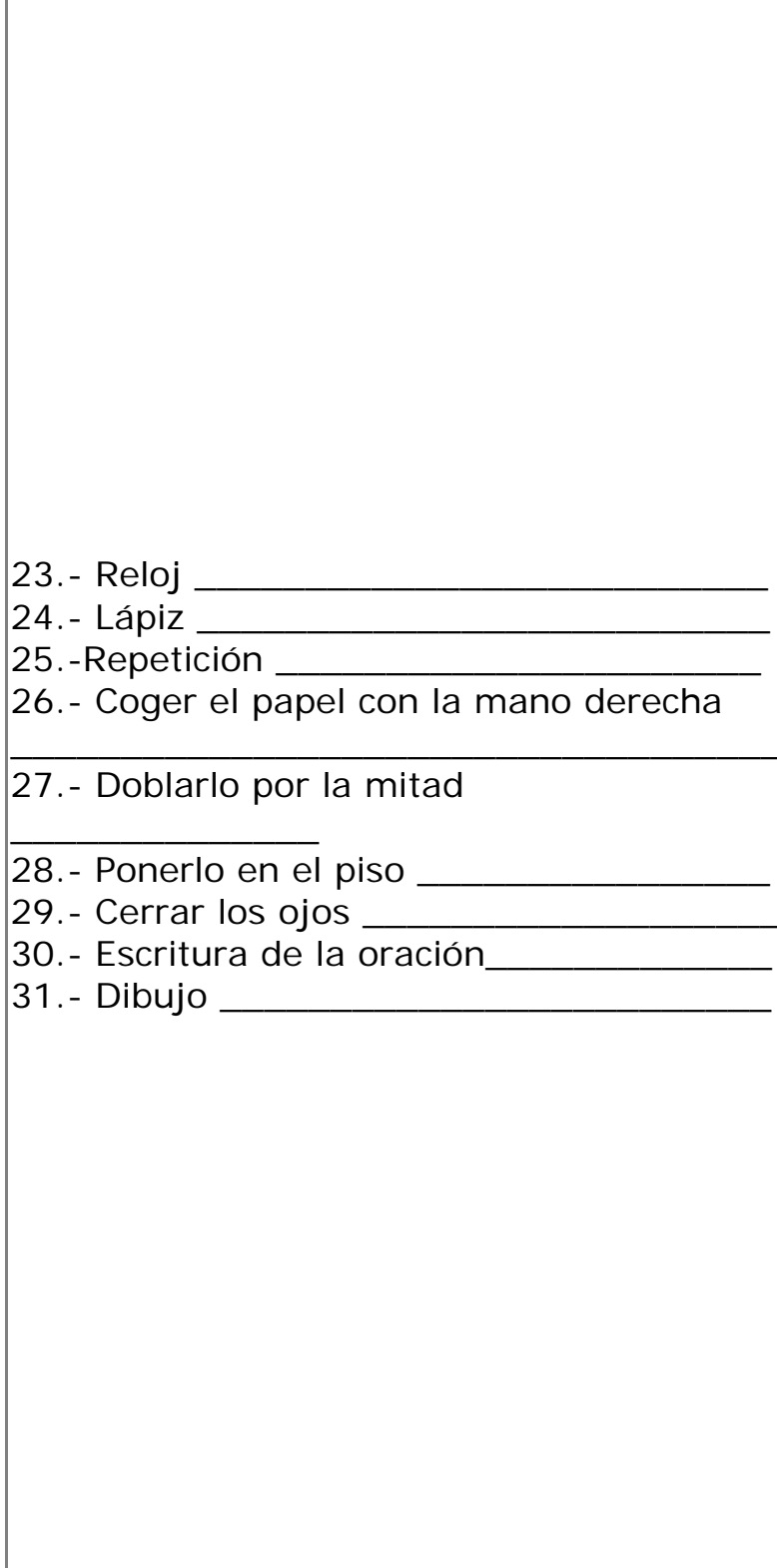 \\
\hline
\end{tabular}

Puntuación:

Sumar el número de respuestas correctas.

En las respuestas del aspecto III, incluir los ítems del 14 al 18 ó el ítem 19, pero no ambos.

El máximo de puntos es 30. es 30 . 


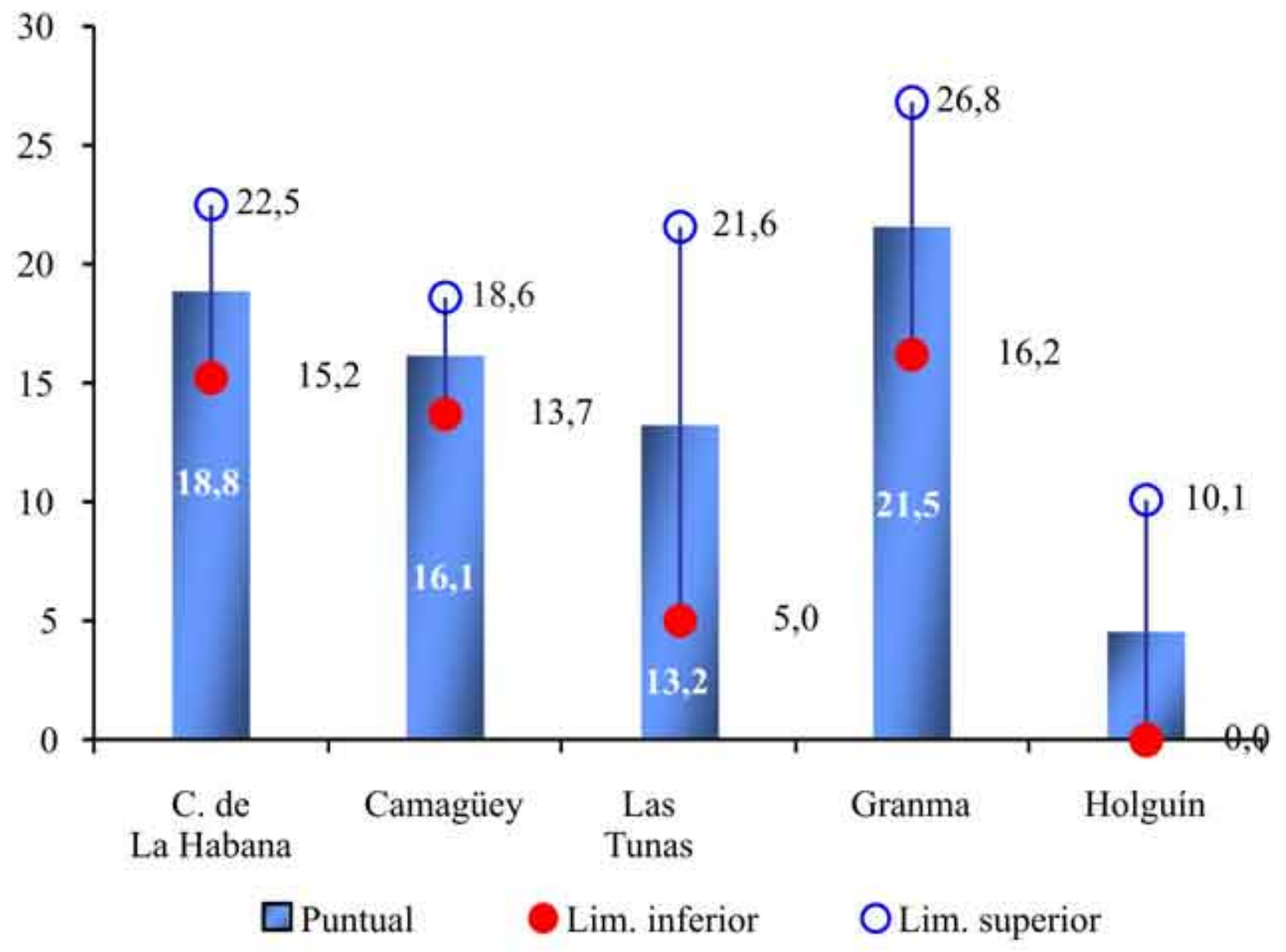

Fuente: tabla 1.

Fig. Tasas de prevalencia de discapacidad mental en adultos mayores por provincia.

Tabla 1. Tasas de discapacidad tipificadas* por edad y sexo según tipo y provincia

\begin{tabular}{|c|c|c|}
\hline \multirow[t]{2}{*}{ Provincia } & \multicolumn{2}{|c|}{ Discapacidad mental } \\
\hline & TDT $_{e}^{*}$ & $\mathrm{TDT}_{3}^{*}$ \\
\hline Ciudad de La Habana & 12,0 & 17,2 \\
\hline Camagüey & 17,5 & 15,6 \\
\hline Las Tunas & 13,2 & 15,2 \\
\hline Granma & 21,6 & 20,2 \\
\hline Holguín & 4,4 & 4,9 \\
\hline
\end{tabular}

*por 100 adultos mayores, TDT : tasa de discapacidad tipificada por edad, TDT : tasa de discapacidad tipificada por sexo. 
Tabla 2. Ancianos con alteraciones del estado mental segín provincia, discapacidad mental y dimensiones del Examen Mínimo del Estado Mental

\begin{tabular}{|c|c|c|c|c|c|c|c|c|c|c|c|}
\hline \multirow[t]{3}{*}{ Provincia } & \multirow{3}{*}{$\begin{array}{l}\text { Discapacidad } \\
\text { mental }\end{array}$} & \multicolumn{10}{|c|}{ Dimensiones del Examen Mínimo del Estado Mental } \\
\hline & & \multicolumn{2}{|c|}{ Orientación } & \multicolumn{2}{|c|}{ Registro } & \multicolumn{2}{|c|}{$\begin{array}{l}\text { Cáculo y } \\
\text { atención }\end{array}$} & \multicolumn{2}{|c|}{ Recuerdo } & \multicolumn{2}{|c|}{ Lenguaje } \\
\hline & & No. & $\%$ & No. & $\%$ & No. & $\%$ & No. & $\%$ & No. & $\%$ \\
\hline \multirow[t]{2}{*}{$\begin{array}{l}\text { Ciudad de La } \\
\text { Habana }\end{array}$} & $\begin{array}{l}\text { Presente } \\
(n=137)\end{array}$ & 106 & 77,3 & 81 & 59,1 & 128 & 93,4 & 120 & 87,6 & 79 & 57,7 \\
\hline & $\begin{array}{l}\text { Ausente } \\
(\mathrm{n}=591)\end{array}$ & 70 & 11,8 & 58 & 9,8 & 261 & 44,1 & 106 & 17,9 & 29 & 4,9 \\
\hline \multirow[t]{2}{*}{ Camagüey } & $\begin{array}{l}\text { Presente } \\
(n=139)\end{array}$ & 115 & 82,7 & 74 & 53,2 & 136 & 97,8 & 131 & 94,2 & 70 & 50,4 \\
\hline & $\begin{array}{l}\text { Ausente } \\
(\mathrm{n}=727)\end{array}$ & 111 & 15,2 & 61 & 8,4 & 339 & 46,6 & 406 & 55,8 & 15 & 2,1 \\
\hline \multirow[t]{2}{*}{ Las Tunas } & $\begin{array}{l}\text { Presente } \\
(n=92)\end{array}$ & 42 & 45,7 & 16 & 17,4 & 83 & 90,2 & 62 & 67,4 & 34 & 37,0 \\
\hline & $\begin{array}{l}\text { Ausente } \\
(n=606)\end{array}$ & 13 & 2,1 & 10 & 1,7 & 189 & 31,2 & 148 & 24,4 & 30 & 5,0 \\
\hline \multirow[t]{2}{*}{ Granma } & $\begin{array}{l}\text { Presente } \\
(n=148)\end{array}$ & 66 & 44,6 & 76 & 51,4 & 96 & 64,9 & 114 & 77,0 & 37 & 25,0 \\
\hline & $\begin{array}{l}\text { Ausente } \\
(\mathrm{n}=540)\end{array}$ & 27 & 5,0 & 21 & 3,9 & 183 & 33,9 & 98 & 18,1 & 49 & 9,1 \\
\hline \multirow[t]{2}{*}{ Holguín } & $\begin{array}{l}\text { Presente } \\
(\mathrm{n}=31)\end{array}$ & 28 & 90,3 & 25 & 80,6 & 29 & 93,5 & 28 & 90,3 & 26 & 83,9 \\
\hline & $\begin{array}{l}\text { Ausente } \\
(\mathrm{n}=661)\end{array}$ & 5 & 0,8 & 1 & 0,2 & 43 & 6,5 & 23 & 3,5 & 3 & 0,5 \\
\hline
\end{tabular}

Tabla 3. Prevalencia de la discapacidad mental en ancianos según provincia y grupos de edad

\begin{tabular}{|l|l|l|l|l|l|l|l|l|l|}
\hline \multirow{2}{*}{ Provincia } & \multicolumn{9}{|c|}{$60-6900$ Grupo de edad } \\
\cline { 2 - 11 } & No. & Tasa & IC $(95 \%)$ & No. & Tasa & IC (95\%) & No. & Tasa * & IC (95\%) \\
\hline $\begin{array}{l}\text { Ciudad de La } \\
\text { Habana }\end{array}$ & 18 & 5,2 & 3,$8 ; 6,6$ & 36 & 16,3 & 8,$5 ; 24,1$ & 83 & 51,2 & 40,$4 ; 62,0$ \\
\hline Camagüey & 19 & 4,8 & 2,$7 ; 6,9$ & 39 & 12,5 & 8,$8 ; 16,2$ & 81 & 50,0 & 42,$3 ; 57,7$ \\
\hline Las Tunas & 22 & 5,8 & 1,$9 ; 9,6$ & 33 & 14,5 & 3,$8 ; 25,2$ & 37 & 42,0 & 9,$5 ; 74,6$ \\
\hline Granma & 23 & 7,2 & 2,$7 ; 11,7$ & 44 & 19,6 & 12,$6 ; 26,5$ & 81 & 56,3 & 45,$8 ; 66,7$ \\
\hline Holguín & 1 & 0,3 & 0,$0 ; 0,8$ & 9 & 4,6 & 0,$0 ; 11,0$ & 21 & 18,1 & 0,$6 ; 35,7$ \\
\hline
\end{tabular}

*por 100 adultos mayores. Su cálculo se realizó tomando como deno minador el total de ancianos de cada grupo de edad y provincia. 
Tabla 4. Prevalencia de discapacidad mental en ancianos segín provincia y sexo

\begin{tabular}{|c|c|c|c|c|c|c|}
\hline \multirow[t]{3}{*}{ Provincia } & \multicolumn{6}{|c|}{ Sexo } \\
\hline & \multicolumn{3}{|c|}{ Masculino } & \multicolumn{3}{|c|}{ Femenino } \\
\hline & No. & Tasa ${ }^{\circ}$ & $\mathrm{IC}(95 \%)$ & No. & Tasa ${ }^{\circ}$ & $\mathrm{IC}(95 \%)$ \\
\hline Ciudad de La Habana & 40 & 15,1 & 11,$2 ; 19,0$ & 97 & 21,0 & 16,$6 ; 25,3$ \\
\hline Camagüey & 44 & 9,6 & 8,$6 ; 10,6$ & 95 & 23,2 & 19,$1 ; 27,3$ \\
\hline Las Tunas & 34 & 12,7 & 10,$2 ; 15,3$ & 58 & 13,5 & 1,$3 ; 25,6$ \\
\hline Granma & 60 & 19,7 & 11,$6 ; 27,7$ & 88 & 23,0 & 18,$7 ; 27,3$ \\
\hline Holguín & 7 & 2,5 & 0,$9 ; 4,1$ & 24 & 5,8 & 0,$0 ; 14,2$ \\
\hline
\end{tabular}

* por 100 adultos mayores. Su cálculo se realizó tomando como deno minador el total de ancianos de cada se xo y provincia.

Tabla 5. Prevalencia de discapacidad mental en ancianos según provincia y ocupación

\begin{tabular}{|l|l|l|l|l|l|l|}
\hline \multirow{2}{*}{ Provincia } & \multicolumn{6}{|c|}{ Ocupación } \\
\cline { 2 - 8 } & \multicolumn{4}{|c|}{ Ocupado } & \multicolumn{4}{c|}{ Desocupado } \\
\cline { 2 - 8 } & No. & Tasa & IC $(95 \%)$ & No. & Tasa & IC (95\%) \\
\hline Ciudad de La Habana & 5 & 7,6 & 1,$5 ; 13,6$ & 132 & 19,9 & 16,$4 ; 3,5$ \\
\hline Camagüey & 13 & 10,8 & 5,$2 ; 16,4$ & 126 & 16,9 & 14,$2 ; 19,6$ \\
\hline Las Turas & 6 & 5,1 & 1,$9 ; 8,2$ & 86 & 14,8 & 5,$1 ; 24,5$ \\
\hline Granma & 14 & 19,2 & 2,$4 ; 35,9$ & 134 & 21,8 & 17,$8 ; 25,8$ \\
\hline Holguín & 0 & 0,0 & - & 31 & 5,1 & 0,$0 ; 10,1$ \\
\hline
\end{tabular}

* por 100 adultos mayores. Su cálculo se realizó tomando como deno minador el total de ancianos de cada se xo y provincia. 
Tabla 6. Prevalencia de discapacidad mental en ancianos según escolaridad y provincia

\begin{tabular}{|c|c|c|c|c|c|c|c|c|c|c|c|c|c|c|c|}
\hline \multirow[b]{3}{*}{ Escolaridad } & \multicolumn{15}{|c|}{ Provincia } \\
\hline & \multicolumn{3}{|c|}{ Ciudad de La Habana } & \multicolumn{3}{|c|}{ Camagïey } & \multicolumn{3}{|c|}{ Las Turas } & \multicolumn{3}{|c|}{ Granma } & \multicolumn{3}{|c|}{ Holguín } \\
\hline & No. & Tasa* & $\begin{array}{l}\text { IC } \\
(95 \%)\end{array}$ & No. & Tasa* & $\begin{array}{l}\mathrm{IC} \\
(95 \%)\end{array}$ & No. & Tasa * & $\begin{array}{l}\mathrm{IC} \\
(95 \%)\end{array}$ & No. & Tasa * & $\begin{array}{l}\text { IC } \\
(95 \%)\end{array}$ & No. & Tasa* & $\begin{array}{l}\text { IC } \\
(95 \%)\end{array}$ \\
\hline Analfabeto & 22 & 66,1 & $\begin{array}{l}49,6 \\
83,7\end{array}$ & 30 & 61,2 & $\begin{array}{l}47,6 \\
74,8\end{array}$ & 17 & 68,0 & $\begin{array}{l}64,2 \\
71,8\end{array}$ & 26 & 72,2 & $\begin{array}{l}62,8 \\
81,7\end{array}$ & 9 & 28,1 & $\begin{array}{l}3,3 \\
52,9\end{array}$ \\
\hline $\begin{array}{l}\text { Primaria } \\
\text { inconclusa }\end{array}$ & 71 & 25,1 & $\begin{array}{l}17,0 \\
33,2\end{array}$ & 34 & 83,7 & $\begin{array}{l}9,4 \\
18,0\end{array}$ & 46 & 31,9 & $\begin{array}{l}7,7 \\
56,2\end{array}$ & 71 & 46,4 & $\begin{array}{l}22,2 \\
70,6\end{array}$ & 12 & 6,5 & $\begin{array}{l}0,0 \\
14,3\end{array}$ \\
\hline $\begin{array}{l}\text { Primaria } \\
\text { concluida }\end{array}$ & 29 & 10,8 & $\begin{array}{l}8,7 \\
12,9\end{array}$ & 43 & 26,9 & $\begin{array}{l}20,0 \\
33,8\end{array}$ & 16 & 7,9 & $\begin{array}{l}0,0 \\
18,2\end{array}$ & 36 & 18,6 & $\begin{array}{l}12,7 \\
24,5\end{array}$ & 6 & 2,9 & $\begin{array}{l}0,0 \\
7,2\end{array}$ \\
\hline $\begin{array}{l}\text { Secundaria } \\
\text { concluida }\end{array}$ & 12 & 14,1 & $\begin{array}{l}7,4 \\
20,9\end{array}$ & 24 & 13,7 & $\begin{array}{l}8,6 \\
18,8\end{array}$ & 4 & 2,9 & $\begin{array}{l}0,3 \\
5,5\end{array}$ & 23 & 10,7 & $\begin{array}{l}7,2 \\
14,2\end{array}$ & 1 & 0,8 & $\begin{array}{l}0,0 \\
2,3\end{array}$ \\
\hline $\begin{array}{l}\text { Preuriversitario } \\
\text { concluido }\end{array}$ & 3 & 8,3 & $\begin{array}{l}2,3 \\
14,4\end{array}$ & 6 & 3,5 & $\begin{array}{l}0,8 \\
6,2\end{array}$ & 9 & 7,7 & $\begin{array}{l}0,0 \\
20,7\end{array}$ & 11 & 9,3 & $\begin{array}{l}4,2 \\
14,4\end{array}$ & 3 & 3,0 & $\begin{array}{l}0,5 \\
5,5\end{array}$ \\
\hline $\begin{array}{l}\text { Universidad } \\
\text { concluida }\end{array}$ & 0 & 0,0 & - & 2 & 3,3 & $\begin{array}{l}0,0 ; \\
7,8\end{array}$ & 0 & 0,0 & - & 2 & 7,1 & $\begin{array}{l}2,4 \\
47,3\end{array}$ & 0 & 0,0 & - \\
\hline
\end{tabular}

* por 100 adultos mayores. Su cálculo se realizó tomando como deno minador el total de ancianos de cada escolaridad y provincia. 